\title{
MORE CHARACTERIZATIONS OF $H$-CLOSED SPACES
}

\author{
JAMES E. JOSEPH
}

AbSTRACT. Several characterizations of $\boldsymbol{H}$-closed spaces are given along with some relating properties and examples.

1. Introduction. Recently, several new characterizations of $H$-closed topological spaces have been given in [2] and [3].

In this article, we introduce the notion of strong-closed subset of a product of two topological spaces and obtain several other characterizations of $H$-closed spaces. In the remainder of this paper, topological spaces will merely be called spaces, $\mathrm{cl}[K]$ will denote the closure of a subset $K$ of a space, and $\operatorname{ad} \nabla$ will denote the adherence of a filterbase $\nabla$ on a space. If $\psi$ and $\lambda$ are functions from a set $X$ to a set $Y$, we will denote $\{x \in X: \psi(x)=\lambda(x)\}$ by $\mathcal{E}(\psi, \lambda, X, Y)$. In the last section we give some examples.

2. Preliminaries. We let $\mathcal{S}$ denote a class of spaces containing as a subclass the Hausdorff completely normal and fully normal spaces. $G(\psi)$ will represent the graph of a function $\psi$.

2.1. Definition [5]. A point $x$ in a space is in the $\theta$-closure of a subset $K$ of the space $(x \in \theta-\operatorname{cl}[K])$ if every open set $V$ about $x$ satisfies $K \cap \operatorname{cl}[V] \neq \varnothing$.

2.2. Definition [5]. A point $x$ in a space is in the $\theta$-adherence of a filterbase $\nabla$ on the space $(x \in \theta-\mathrm{ad} \nabla)$ if $x \in \theta-\operatorname{cl}[F]$ for each $F \in \nabla$. A filterbase $\nabla$ $\theta$-converges to $x\left(\nabla \rightarrow{ }_{\theta} x\right)$ if for each $V$ open about $x$, there is an $F \in \nabla$ with $F \subset \operatorname{cl}[V]$.

2.3. Definition. If $X$ and $Y$ are spaces and $K \subset X \times Y$, we will say that $(x, y) \in X \times Y$ is in the strong-first-coordinate-closure (strong-second-coordinate-closure $)$ of $K$ if $K \cap(\operatorname{cl}[V] \times W) \neq \varnothing(K \cap(V \times \operatorname{cl}[W]) \neq \varnothing)$ whenever $V$ is open about $x$ and $W$ is open about $y$. In this case, we will use the notation $(x, y) \in(1) \theta-\operatorname{cl}[K]((x, y) \in(2) \theta-\operatorname{cl}[K])$. If $K=(1) \theta-\operatorname{cl}[K](K=$ (2) $\theta-\mathrm{cl}[K])$ we will say that $K$ is strong-first-coordinate-closed (strong-secondcoordinate-closed). In this case, we will say that $K$ is (1) $\theta$-closed ((2) $\theta$-closed).

2.4. Definition [2]. A function $\psi: X \rightarrow Y$ has a strongly-closed graph if for each $(x, y) \in(X \times Y)-G(\psi)$, there are open sets $V \subset X, W \subset Y$ about $x$ and $y$, respectively, satisfying $(V \times(\operatorname{cl}[W])) \cap G(\psi)=\varnothing$.

We utilize the following characterization of functions with strongly-closed graphs into Hausdorff spaces. The proof is omitted.

Received by the editors June 21, 1976 and, in revised form, September 16, 1976.

AMS (MOS) subject classifications (1970). Primary 54D20.

Key words and phrases. $H$-closed spaces, graphs, projections. 
2.5. THEOREM. A function $\psi$ into a Hausdorff space $Y$ has a strongly-closed graph if and only if $\theta-\operatorname{ad} \psi(\nabla) \cup\{\psi(x)\}=\{\psi(x)\}$ for each $x \in X$ and filterbase $\nabla$ on $X-\{x\}$ with $\nabla \rightarrow x$.

We note that a function $\psi: X \rightarrow Y$ has a strongly-closed graph if and only if $G(\psi)$ is a (2) $\theta$-closed subset of $X \times Y$.

2.6. Definition [4]. A function $\psi$ from a space $X$ to a space $Y$ is weakly-continuous if for each $x \in X$ and $W$ open in $Y$ about $\psi(x)$, there is a $V$ open in $X$ about $x$ satisfying $\psi(V) \subset \operatorname{cl}[W]$.

We present the following characterization of weakly-continuous functions. We omit the proof.

2.7. TheOREM. A function $\psi: X \rightarrow Y$ is weakly continuous if and only if $\psi(\nabla) \rightarrow_{\theta} \psi(x)$ for each filterbase $\nabla$ on $X$ such that $\nabla \rightarrow x$.

2.8. Definition. Let $X$ be a set, let $x_{0} \in X$, and let $\nabla$ be a filterbase on $X$; $\left\{A \subset X: x_{0} \in X-A\right.$ or $F \cup\left\{x_{0}\right\} \subset A$ for some $\left.F \in \nabla\right\}$ is a topology on $X$ which will be called the topology on $X$ associated with $x_{0}$ and $\nabla . X$ equipped with this topology will be called the space associated with $x_{0}$ and $\nabla$. We will denote this space by $X\left(x_{0}, \nabla\right)$.

The following readily established theorem is used in the sequel.

2.9. TheOREM. Let $X$ be a set, let $x_{0} \in X$ and let $\nabla$ be a filterbase on $X$ which has empty intersection on $X-\left\{x_{0}\right\}$. Then $X\left(x_{0}, \nabla\right)$ is in class $\delta$.

3. Characterizations of $H$-closed spaces via graphs. We employ as a primitive the following characterization of $H$-closed spaces from [5].

3.1. Definition. A Hausdorff space is $H$-closed if each filterbase on the space has a nonempty $\theta$-adherence.

We will make use of the following theorem in the sequel.

3.2. TheOREM. A Hausdorff space is $H$-closed if and only if each filterbase on the space with at most one $\theta$-adherent point $\theta$-converges.

Proof. Necessity. Let $\nabla$ be a filterbase on the $H$-closed space $X$, let $x_{0} \in X$ and suppose that $\theta-\operatorname{ad} \nabla \cap\left(X-\left\{x_{0}\right\}\right)=\varnothing$. Then $\theta-\operatorname{ad} \nabla=\left\{x_{0}\right\}$. Now, let $V$ be open about $x_{0}$ and suppose that each $F \in \nabla$ satisfies $F \cap(X-\operatorname{cl}[V]) \neq$ $\varnothing$. Since $\{F \cap[X-\operatorname{cl}[V]]: F \in \nabla\}$ is a filterbase on the $H$-closed space $X$,

$$
\begin{aligned}
\varnothing & \neq \bigcap_{\nabla} \theta-\operatorname{cl}[F \cap(X-\operatorname{cl}[V])] \subset \theta-\operatorname{ad} \nabla \cap \theta-\operatorname{cl}[X-\operatorname{cl}[V]] \\
& =\left\{x_{0}\right\} \cap \operatorname{cl}[X-\operatorname{cl}[V]] \subset\left\{x_{0}\right\} \cap(X-V)=\varnothing,
\end{aligned}
$$

which is a contradiction.

Sufficiency. Let $\nabla$ be a filterbase on $X$ and suppose that $\theta-\operatorname{ad} \nabla \cap(X-$ $\left.\left\{x_{0}\right\}\right)=\varnothing$. Then $\nabla \rightarrow_{\theta} x_{0}$.

The proof is complete.

In what follows, $\pi_{x}: X \times Y \rightarrow X$ and $\pi_{y}: X \times Y \rightarrow Y$ represent the projections. 
3.3. THEOREM. If $\psi: X \rightarrow Y$ is weakly-continuous and $K$ is a (2) $\theta$-closed subset of $X \times Y$, then $\pi_{x}(K \cap G(\psi))$ is closed in $X$.

Proof. Let $K$ be a (2) $\theta$-closed subset of $X \times Y$, let $v \in \operatorname{cl}\left[\pi_{x}(K \cap G(\psi))\right]$, let $V$ be open about $v$, and let $W$ be open about $\psi(v)$. There is an $A$ open about $v$ satisfying $\psi(A) \subset \operatorname{cl}[W]$ and $(A \cap V) \cap \pi_{x}(K \cap G(\psi)) \neq \varnothing$. Let $z \in(A \cap V) \cap \pi_{x}(K \cap G(\psi))$. Then $(z, \psi(z)) \in K \cap(V \times \operatorname{cl}[W])$. This establishes that $(v, \psi(v)) \in(2) \theta-\operatorname{cl}[K]=K$ and that $v \in \pi_{x}(K \cap G(\psi))$; the proof is complete.

3.4. Corollary. If $\psi: X \rightarrow Y$ has a strongly-closed graph and $\psi: X \rightarrow Y$ is weakly-continuous, then $\mathcal{E}(\psi, \lambda, X, Y)$ is closed in $X$.

Proof. $G(\psi)$ is (2) $\theta$-closed and $\mathcal{E}(\psi, \lambda, X, Y)=\pi_{x}(G(\psi) \cap G(\lambda))$.

3.5. Theorem. A Hausdorff space $Y$ is $H$-closed if and only if for any space $X$ in class $\mathcal{S}$ and bijections $\psi, \lambda: X \rightarrow Y$ with strongly-closed graphs, $\mathcal{E}(\psi, \lambda, X, Y)$ is closed in $X$.

Proof. Strong necessity. Let $Y$ be an $H$-closed space, let $X$ be any space and let $\psi, \lambda: X \rightarrow Y$ be any functions with strongly-closed graphs. From the main result in [2], $\psi$ and $\lambda$ are weakly-continuous. Thus, $\mathcal{E}(\psi, \lambda, X, Y)$ is closed from Corollary 3.4 .

Sufficiency. Let $\nabla$ be a filterbase on the Hausdorff space $Y$, let $y_{0} \in Y$ and suppose $\theta-\operatorname{ad} \nabla \cap\left(Y-\left\{y_{0}\right\}\right)=\varnothing$. If $\nabla \nrightarrow_{\theta} y_{0}$, then there is a $V_{0}$ open about $y_{0}$ such that $\nabla_{1}=\left\{F \cap\left(Y-\operatorname{cl}\left[V_{0}\right]\right): F \in \nabla\right\}$ is a filterbase on $Y$. Choose $x_{0} \in Y-\operatorname{cl}\left[V_{0}\right]$ and define $\psi, \lambda: Y\left(y_{0}, \nabla_{1}\right) \rightarrow Y$ as follows: $\psi(x)=x$ for all $x ; \lambda\left(x_{0}\right)=y_{0}, \lambda\left(y_{0}\right)=x_{0}$ and $\lambda(x)=x$ otherwise. Then $\mathcal{E}\left(\psi, \lambda, Y\left(y_{0}, \nabla_{1}\right), Y\right)=Y-\left\{x_{0}, y_{0}\right\}$, which is not closed in $Y\left(y_{0}, \nabla\right)$. We see that $\psi$ and $\lambda$ are bijections and we show that $\psi$ and $\lambda$ have strongly-closed graphs to complete the proof since $Y$ does not satisfy the required condition.

(a) $G(\psi)$ is strongly-closed. Let $y \in Y\left(y_{0}, \nabla_{1}\right)$ and let $\nabla_{2}$ be a filterbase on $Y\left(y_{0}, \nabla_{1}\right)-\{y\}$ with $\nabla_{2} \rightarrow y$. Then $y=y_{0}$ and for each $F \in \nabla$, there is an $F_{2} \in \nabla_{2}$ satisfying $F_{2} \subset F$. Thus, $\theta-\operatorname{ad} \psi\left(\nabla_{2}\right) \subset \theta-\operatorname{ad} \psi(\nabla)=\theta-\operatorname{ad} \nabla$. This shows that $\psi$ has a strongly-closed graph by Theorem 2.5 .

(b) $G(\lambda)$ is strongly-closed. Let $y \in Y\left(y_{0}, \nabla_{1}\right)$ and let $\nabla_{2}$ be a filterbase on $Y\left(y_{0}, \nabla_{1}\right)-\{y\}$ with $\nabla_{2} \rightarrow y$. Then $y=y_{0}$ and for each $F \in \nabla$, there is an $F_{2} \in \nabla_{2}$ satisfying $F_{2} \subset F \cap\left(Y-\operatorname{cl}\left[V_{0}\right]\right)$. So $\theta-\operatorname{ad} \lambda\left(\nabla_{2}\right)=\theta-\operatorname{ad} \nabla_{2} \subset \theta-\operatorname{ad} \nabla_{1}$ $=\varnothing$. So, $\lambda$ has a strongly-closed graph by Theorem 2.5 .

The proof is complete.

3.6. THEOREM. A Hausdorff space $Y$ is $H$-closed if and only if for any space $X$ in class $\mathcal{S}$ and functions $\psi, \lambda: X \rightarrow Y$ (one a bijection) with strongly-closed graphs, $\mathcal{E}(\psi, \lambda, X, Y)=X$ whenever $\mathcal{E}(\psi, \lambda, X, Y)$ is dense in $X$.

Proof. Strong necessity. Let $Y$ be an $H$-closed space, let $X$ be any space and let $\psi, \lambda: X \rightarrow Y$ be any functions with strongly-closed graphs. From 
Theorem 3.5, $\mathcal{E}(\psi, \lambda, X, Y)$ is closed in $X$; so $\mathcal{E}(\psi, \lambda, X, Y)=X$ if $\mathcal{E}(\psi, \lambda, X, Y)$ is dense in $X$.

Sufficiency. We follow the proof of the sufficiency of Theorem 3.5 to the point immediately preceding the definition of $\lambda$. We define $\lambda: Y\left(y_{0}, \nabla_{1}\right) \rightarrow Y$ by $\lambda\left(y_{0}\right)=x_{0}$ and $\lambda(x)=x$, otherwise. Then $\delta\left(\psi, \lambda, Y\left(y_{0}, \nabla_{1}\right), Y\right)=Y-$ $\left\{y_{0}\right\}$, which is dense in $Y\left(y_{0}, \nabla_{1}\right)$. By arguments similar to those in the proof of the sufficiency of Theorem 3.5, we can show that $G(\psi)$ and $G(\lambda)$ are strongly-closed.

The proof is then complete.

4. A characterization of $H$-closed spaces via projections. In [3], the following theorem has been proved.

4.1. TheOREM. A Hausdorff space $X$ is $H$-closed if and only if $\pi_{y}: X \times Y \rightarrow$ $Y$ maps $\theta$-closed subsets onto $\theta$-closed subsets of $Y$ for every space $Y$.

We offer the following similar characterization.

4.2. Theorem. A Hausdorff space $X$ is $H$-closed if and only if the projection $\pi_{y}: X \times Y \rightarrow Y$ maps (1) $\theta$-closed subsets onto closed subsets for every space $Y$ in class $\delta$.

Proof. Strong necessity. Let $X$ be $H$-closed, let $Y$ be any space and let $K \subset X \times Y$ be (1) $\theta$-closed. If $y \in \operatorname{cl}\left[\pi_{y}(K)\right]$, then $\nabla=\left\{\pi_{x}(K \cap(X \times V))\right.$ : $V$ open about $y\}$ is a filterbase on $X$. There is an $x \in \theta-\operatorname{ad} \nabla$. It is not difficult to show that $(x, y) \in(1) \theta-\operatorname{cl}[K]=K$ and, consequently, that $y \in$ $\pi_{y}(K)$.

Sufficiency. Let $\nabla$ be a filterbase on the Hausdorff space $X$ and suppose $\theta-\operatorname{ad} \nabla=\varnothing$. Let $y_{0} \notin X$ and let $Y=X \cup\left\{y_{0}\right\}$. Let $K=\{(x, x): x \in X\}$. We show that $K$ is a (1) $\theta$-closed subset of $X \times Y\left(y_{0}, \nabla\right)$. Let $(x, y) \in(X \times$ $Y)-K$. Then $x \neq y_{0}$ and $x \neq y$; so there is a $V$ open about $x$ and $F \in \nabla$ satisfying $F \cup\left\{y_{0}, y\right\} \subset X-\operatorname{cl}[V]$. Since $F \cup\left\{y_{0}, y\right\}$ is open about $y$ in $Y\left(y_{0}, \nabla\right)$ and $\left(\operatorname{cl}[V] \times\left(F \cup\left\{y, y_{0}\right\}\right)\right) \cap K=\varnothing$, we have $(x, y) \notin(1) \theta$ $\operatorname{cl}[K]$; thus, $K$ is (1) $\theta$-closed. This then forces $\pi_{y}(K)$ to be closed in $Y\left(y_{0}, \nabla\right)$ : this cannot happen because $y_{0} \in \operatorname{cl}\left[\pi_{y}(K)\right]-\pi_{y}(K)$.

The proof is complete.

5. Some examples. In this section, we give examples to indicate some of the limitations on the weakening of hypotheses in the theorems in this article. Let $N$ be the set of positive integers and for each $k \in N$, we let $E(k)=\{k+$ 1/n: $n \in N\}$. Let $Y=\{0\} \cup N \cup \cup_{k \in N} E(k)$ with the topology generated by $\{V \cap(Y-\{0\}): V$ is a usual open subset of the reals $\} \cup\{\{0\} \cup$ $\left.\cup_{k \geqslant m} E(k): m \in N\right\}$ as base. Let $Z(k)=\{n \in N: n \geqslant k\}$ and let $\nabla=$ $\{Z(k): k \in N\}$. $Y$ is known to be $H$-closed [1].

5.1. EXAMPLES. No "strongly-closed graph" requirement can be replaced by a "closed graph" requirement in either of Theorems 3.5 or 3.6. Let $\psi: Y(0, \nabla) \rightarrow$ $Y$ be the identity and let $\lambda, \alpha: Y(0, \nabla) \rightarrow Y$ be defined by $\lambda(1)=0, \lambda(0)=1$ and $\lambda(x)=x$ otherwise, $\alpha(0)=1$ and $\alpha(x)=x$ otherwise. Then $\psi$ has a 
strongly-closed graph while $\lambda$ and $\alpha$ both have closed graphs. However, $\mathcal{E}(\psi, \lambda, Y(0, \nabla), Y)=Y-\{0,1\}$, which is not closed in $Y(0, \nabla)$. Furthermore, $\mathcal{E}(\psi, \alpha, Y(0, \nabla), Y)=Y-\{0\}$, which is dense in $Y(0, \nabla)$. Now, if $\psi$ : $Y(1, \nabla) \rightarrow Y$ is the identity function and $\alpha: Y(1, \nabla) \rightarrow Y$ is defined by $\alpha(1)=0, \alpha(x)=x$ otherwise, we see that $\psi$ has a closed graph, $\alpha$ has a strongly-closed graph, and $\mathcal{E}(\psi, \alpha, Y(1, \nabla), Y)=Y-\{1\}$, which is dense in $Y(1, \nabla)$.

\section{REFERENCES}

1. M. P. Berri, J. P. Porter and R. M. Stephenson, Jr., A survey of minimal topological spaces, General Topology and its Relations to Modern Analysis and Algebra, III (Proc. Conf., Kanpur, 1968), Academia, Prague, 1971, pp. 93-114. MR 43 \#3985.

2. L. L. Herrington and P. E. Long, Characterizations of H-closed spaces, Proc. Amer. Math. Soc. 48 (1975), 469-475. MR 51 \# 1737.

3. J. E. Joseph, On H-closed spaces, Proc. Amer. Math. Soc. 55 (1976), 223-226.

4. Norman Levine, $A$ decomposition of continuity in topological spaces, Amer. Math. Monthly 68 (1961), 44-46. MR 23 \# A3548.

5. N. V. Veličko, H-closed topological spaces, Mat. Sb. 70(112) (1966), 98-112; English transl., Amer. Math. Soc. Transl. (2) 78 (1968), 103-118. MR 33 \#6576.

Department of Mathematics, Federal City College, Washington, D. C. 20001

Current address: Department of Mathematics, Howard University, Washington, D. C. 20059 